ing compression of nerve root itself or of vascular system or of covering membrane of that root. The writer demonstrated X-ray pictures of initial change with minute spur at the Luschka joint and also of moderately advanced and far advanced.

\title{
14. Study of Posttraumatic Chronic Headache, Part I.
}

\author{
Masashichi Kawano, Kazuo Kitajima, Ryusuke Nishimura, \\ Hidenobu Oshibuchi and Goichi Matsumura \\ Second Department of Surgery, Nagasaki University School of Medicine
}

Headache is the commonest symptom of posttraumatic syndrome. This posttraumatic headeche has a certain characteriscs, (1) it tends to become chronic, (2) it does not respond well to treatment, (3) it does not correlate to severity of primary injury, (4) it is predominantly in the occipital area of the head, and (5) it is accompanied by dizziness, nausea, fatigability and other complaints.

Pathogenesis of this posttraumatic chronic headache has been subjects of controversy. One blamed the midbrain injury as the cause of autonomic nervous system disturbance, other attributed to neurosis, and still other to circulatory disorder of the vertebral artery. However, it has been pointed out by some authors that tenderness of the great occipital nerve was found in many patients of posttraumatic chronic headache which responds well to the nerve block. Many factors can contribute to the development of neuralgia of this nerve, but hypoxia of the scalp due to long bed rest and the use of ice pillow could be one of the avoidable causes.

In our investigation of 300 head injury patients who visited our clinic in $1965,34.6$ per cent or 47 cases complained of chrochic headache, and tenderness was proved in 45.1 per cent of these headache patients. Investigation also revealed that 85 per cent of posttraumatic headche patients have cooled their head for various length of time, the longest being 83 days.

Electric current stimulation test was done on 21 headache patients and 10 controls. As the results, the threshold of sensitivity to electric current is lowered in the posttraumatic headache patients with tenderness of the great occipital nerve, whereas the threshold of pain sensation is elevated and radiated pain is felt as compared with the controls. This finding sug- 
gests the presence of some change in th great occipital nerve tissues.

When the scalp of rabbit was cooled with ice bag as cold as 2 to $4^{\circ} \mathrm{C}$, no significant change is observed in shorter period, but some changes appear after $2 \frac{1}{2}$ hours of cooling and becomes evident in 7 hour cooling. Those changes include swelling of mitochondria, segmentation of cristae and enlargment of axon-Schwann interface. From these clinical and experimental studies it is assumed that cooling of the scalp for unduly prolonged period, a common practice in Japan, will create an untoward influence and damage to the great occipital nerve leading to neuralgia manifested as chronic occipital headache. Therefore, the use of ice pillow and long bed rest should be discouraged. As treatment of this type headache we found nerve block with procaine and Solcoceryl combination was more effective than procaine alone or combination with vitamin $B_{1}$ product.

\title{
15. Injuries Involving the Nose, Sinuses and Anterior Cranial Fossa and Liquorrhea
}

\author{
Osamu IIDA and Ryo TAKAHASHI \\ ENT Dept., The Jikei University School of Medicine
}

A severe injuries to the middle third of the face may be associated with the fractures of the frontal sinus, ethmoidal labyrinth and sphenoidal sinus and sometimes might occur cerebrospinal rhinorrhea.

Nine such cases are repoted. Three of them are caused by various trauma and six cases are by traffic accident.

Six cases are treated by frontal sinus operation and lateral rhinotomy is performed in three cases.

The locality of the dural tears are confirmed in eight cases, of which four cases are repaired by means of fascia graft.

It is of deep significance that by sinusitis intracranial complication might occur in these cases and for this reason we believe that one should take up a positive attitude to manage the chronic inflammation in the nose and sinuses.

Lateral rhinotomy is useful procedure not only to remove the sinusitis but also to repair the dural tear without craniotomy. 